\title{
Exploring Urban Events with Transitory Search on Mobiles
}

$\begin{array}{ll}\text { Per M. Nielsen } & \text { Jon Pearce } \\ \text { Dept. Computer Science } & \begin{array}{l}\text { Dept. Comp. and inf. Systems } \\ \text { Aalborg University }\end{array} \\ \begin{array}{l}\text { Denmark } \\ \text { pmni10@student.aau.dk }\end{array} & \begin{array}{l}\text { Australia } \\ \text { j.pearce@unimelb.edu.au }\end{array} \\ \text { Jeni Paay } & \text { Jesper Kjeldskov } \\ \text { Dept. Computer Science } & \text { Dept. Computer Science } \\ \text { Aalborg University } & \text { Aalborg University } \\ \text { Denmark } & \text { Denmark } \\ \text { jeni@cs.aau.dk } & \text { jesper@cs.aau.dk }\end{array}$

Permission to make digital or hard copies of part or all of this work for personal or classroom use is granted without fee provided that copies are not made or distributed for profit or commercial advantage and that copies bear this notice and the full citation on the first page. Copyrights for third-party components of this work must be honored. For all other uses, contact the Owner/Author.

Copyright is held by the owner/author(s).

MobileHCI '15 Adjunct, August 25-28, 2015, Copenhagen, Denmark ACM $978-1-4503-3653-6 / 15 / 08$

http://dx.doi.org/10.1145/2786567.2793692

\begin{abstract}
Searching for unknown urban events in the hope of making serendipitous discoveries has been the focus of multiple mobile tourist guide and recommender systems. This study investigates how transitory search can be used as an interaction mechanism for making such discoveries on a mobile phone. Our prototype, onMelbourne, returns a changing collection of urban events within physical and temporal proximity based on the setting and adjustment of search sliders by the user. Laboratory tests with 5 participants show that users understand transitory search for urban events and do make serendipitous discoveries. In addition we found that users fear missing out on events not shown by the system, and that the choice of labels on sliders impacts their understanding of a slider set halfway. The contribution of the paper is a better understanding of how transitory search can facilitate explorative behaviour when browsing for urban events on mobiles.
\end{abstract}

\section{Author Keywords}

Exploration; Explorative Search; Transitory Search; Event finding; Sliders; Smartphones; User Interfaces

\section{ACM Classification Keywords}

H.5.m. Information interfaces and presentation (e.g., $\mathrm{HCI})$ : Miscellaneous. 


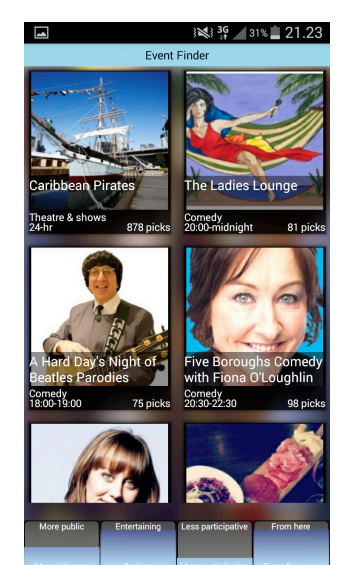

Figure 1. Main screen with sliders and results

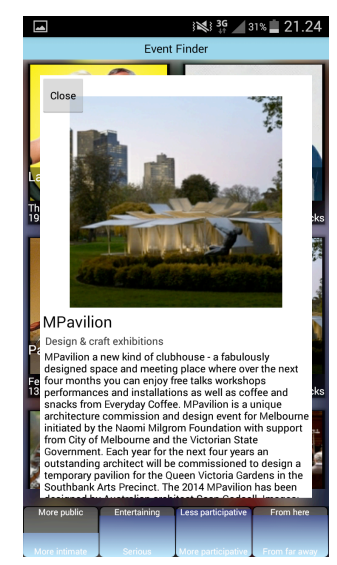

Figure 2. Details of the selected event

\section{Introduction}

Keyword and category search using text based search engines has become a common tool when looking up information online, especially when the search criteria are well defined. But when the search criteria are ill defined and the dataset is large, a more open and explorative, or transitory, approach is required in order to explore and compare results, and reformulate preferences until a satisfactory or interesting result has been returned.

Pearce et al. $[8,9,10,11]$ have worked with exploration of large data sets using the iFish application. iFish is a desktop system, where the user express his/her preferences using sliders and filters causing results to be instantly reordered, so that best matching results appear on top of the list. They investigated how students explore university subjects and found that participants did not explore subjects more deeply than with a printed handbook. Once set, the participants did not alter their sliders and they were not satisfied with seeing only a subset of the subjects available [11]. Later, they conducted a comparative study of a two restaurant finding systems. One system used sliders to indicate preferences, while the other used menus to indicate preferences and a "search" button to start searching. They found the system with sliders worked better when the search parameters were vague and the system with menus was better for welldefined search parameters. Users spent more time reading about the restaurants using the slider system and were more satisfied [8]. iFish has shown the usefulness of setting sliders as search criteria in exploration of different datasets on a desktop computer. However, desktop computers have plenty of screen space to properly show enough information to compare items and manipulate queries simultaneously, while situated in a static use environment unbounded by time and space. Mobile phones do not have this luxury, and it is not known how such an explorative system would work on a mobile device used in the urban context and for data as volatile as urban events.

Building upon the idea of iFish, this paper investigates the concept of exploring data using transitory search for rapid preference reformulation on mobiles. In this type of search users set and adjust sliders while out on the town, retrieving a collection of results visible in the device window that shuffles and reorders dynamically in response to changing search parameters. The top most items represent the best fit events for the combination of preferences set. Search results are therefore ephemeral and transient depending on the interaction of moving sliders to explore content.

\section{Related Work}

A mobile app for serendipitous discovery of events was developed by Forsblom, Nurmi, Åman and Liikkanen [5] that pushes notifications to a user's mobile phone about festival events within geographic proximity. Proximity was the only search criteria in this system and the user did not have to do anything active to get results sent to them. They compared proximity filtering with pushing random events (located within 15mins travel distance) and found that recommendations of events based on nearby location were not rated more relevant than recommendations with a random location. However, Schaller, Harvey and Elsweiler [13] observed the opposite for events distributed over longer distances, as travel time was considered important by users. In addition, they found that when the search criteria were well-defined users used a search query. Conversely, 
when users wanted inspiration they browsed through lists of genres and locations [13]. This behaviour connects well with Marchionini [7] who distinguishes between two types of search activities, look-up search and exploratory search.

Recommender systems (RS) have been used for several years to recommend items such as, e.g., movies, books, and news [1, 6, 12]. Ricci et al. [12] describe six types of RSs, including the constraint- and knowledge-based RS. This RS recommends items based on constraints and rules explicitly defined by the user. Gavalas et al. [6] identify a challenge to this approach, since people often don't know what their preferences are until they are presented with a choice. This makes it difficult for users to give the right starting input to this kind of RS, resulting in non-optimal results. To address this issue, the choice of rules and constraints needs to be dynamic and enable the user to easily explore the results of different constraints and rules to ease decision-making. RSs can also be used with mobile devices, although this presents a challenge for visualizing recommendations due to the limited screen space [6]. Context-aware tourist guides like GUIDE [4], COMPASS [14], and Magitti [3] help tourists visiting new locations, facilitating searches for nearby shops, restaurants, museums, public services, etc. In busy areas the number of search results can be very high, making it difficult to make an informed decision [14].

\section{Transitory Search}

The concept of transitory search is based on the existing concept of transitory information [2]. That is, it is temporary, transient and impermanent. In terms of transitory information, research by Ayers and Youseff [2] is concerned with the type of information contained in educational animations and how this temporality impacts learning of the information content. When thinking about exploratory search [7] with large datasets of information about the world around us, investigation of that information, including discovery and transformation, becomes as equally important an outcome as learning, and can even become the focus, depending on the situation. Transitory search allows for a changing set of outcomes that can "bubble to the top" depending on in-time and in-place adjustment of the degrees of each search criteria. Transitory search can be seen as an interesting alternative to directed search when the domain is leisure, and the outcomes are happily ephemeral, serendipitous and fuzzy.

\section{Case and Prototype System}

As an example case we developed a prototype system for an urban festival in Melbourne, Australia. Like many other large cities, Melbourne hosts many events and festivals during the year. During 2015, the city will host at least 50 festivals and between 70 and 110 events every single day. With that many events on offer, figuring out which event to go to can be a difficult decision-making task. Print material may have limited utility in such situations, since it can take quite a long time to browse through all events, which might not even be available at the current time and place. As our specific case, we started out by focussing on the "Melbourne Festival" in the Melbourne CBD.

Based on contextual interviews and workshops a prototype was developed and evaluated. The prototype was developed for a Samsung Galaxy SIII smartphone in Java on Android, utilizing many of the standard user interface elements and a few custom sliders. 
Data about 485 events was extracted from ThatsMelbourne.com.au and a subset of 80 events stored locally in an SQLite database upon installation. These events were then manually rated and tagged in respect to the different search criteria that were going to be used by the prototype system.

\section{onMelbourne}

The onMelbourne system consists of a main screen and a popup screen (figures $1 \& 2$ ). The main screen is where the primary activity of event exploration takes place, while the popup screen contains additional information about a selected event. The main screen consists of an ordered list of events taking place within the city on the current day. In order to determine the nature and attractiveness of an event, and simultaneously being able to perform comparisons of events, emphasis has been put on displaying relatively large pictures of the events for easy recognition. These pictures are placed on cards in a scrollable grid structure. Each picture has a 1:1 aspect ratio, with a width taking up roughly half of the screen, resulting in approximately 6 cards on display at one time. This way the pictures are large enough for the user to see what it is, yet small enough to fit amongst others for comparison. By displaying multiple events on the screen at the same time, it is also possible to see how the order of events changes as a result of adjusting indicated preferences.

Inspired by ideas from the iFish applications, transitory search is facilitated by four sliders for indicating preferences. The four sliders are located at the bottom of the grid view screen using proportional colour coding to indicate the current setting of the sliders (figure 3 ). Upon tapping one of the sliders, the selected slider expands to cover about $1 / 3$ of the height of the screen enabling easy manipulation and fine grained value setting for that slider (figure 4). Screen space is saved by only showing an expanded slider when it is able to be manipulated. Upon dragging the slider handle, or touching somewhere on the slider, the weight of that slider value is altered, and the events are reordered according to the combined slider setting. When releasing the finger from the screen, the slider retracts and the results list scrolls to the top, showing the events that best match the current slider settings. It is possible to directly see the effect of changing sliders, as the list dynamically reorders (or "bubbles to the top") while the sliders are being manipulated, thus making the activity of searching a transitory and exploratory one. This invites the user to explore the data, through ongoing adjustment of the sliders based on the results.

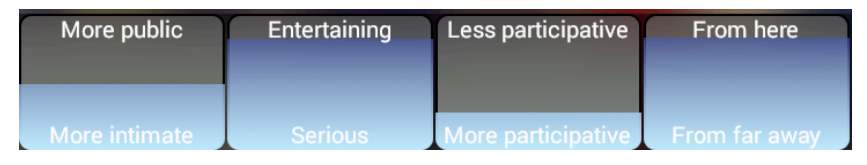

Figure 3. Sliders in non-adjustable states.

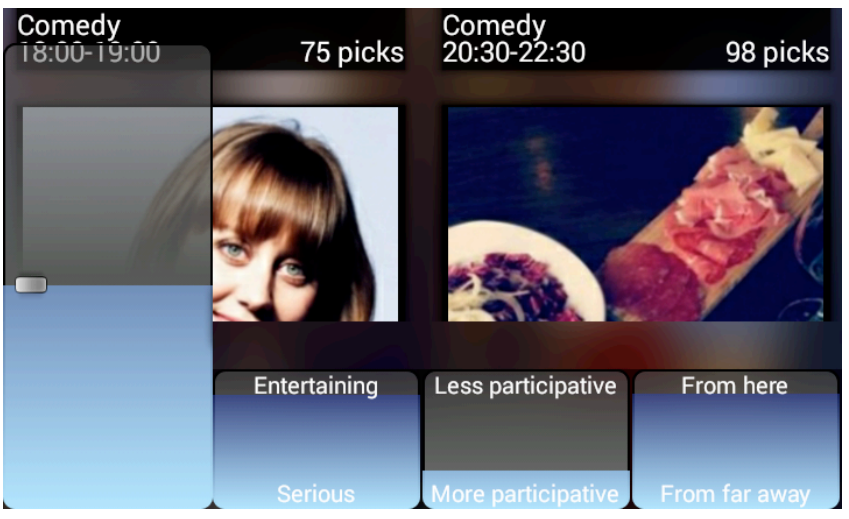


Figure 4. The same sliders, but with the first expanded into an adjustable state, showing slider handle at the half way setting.

In the database, each of the 80 events was manually assigned four integer values from 0-100, categorizing each event. When changing the slider setting, the events that most closely match all the sliders are shown at the top of the list, left to right, in descending order. Assigning these values was done by judging events based on their description, venue and price. Categorization heuristics were also applied, in which categories of events (e.g. comedy, lectures, concerts etc.) were assigned specific ranges of slider values. The pairs of slider labels were chosen through an inductive process applied during a design workshop, and refined using outcomes from the initial contextual interviews. The resulting slider continuums are:

Public $\leftrightarrow$ Intimate: categorizes events based on the size of the venue, amount of visitors and/or atmosphere.

Events could range from street art to cooking classes.

Entertaining $\leftrightarrow$ Serious: differentiates the purpose of the event, for example, comedy shows versus environmental talks.

More participative $\leftrightarrow$ Less participative: Distinguishes events based on how much the visitor is able to participate in the event by using their body and/or mind. This could range from a photography workshop to a cinema movie.

From far away $\leftrightarrow$ From here: indicates what part of the world the event comes from. They tend to be taken as both an indicator of how exotic an event is, and an indicator of the chance of it happening again soon, as well as being used to assess the quality of the event.

\section{Evaluation}

To investigate how useful transitory search is for exploring urban events, a laboratory evaluation of the prototype was conducted in November 2014. Five participants were given the task of finding a comedy event, to understand how they interpret and adjust the sliders to find a specific kind of event. The second task involved finding an event appropriate as an outing for their work colleagues. Lastly, they were asked to find an event they would like to go to themselves, adjusting the sliders according to their own preferences. The focus of the evaluation was on the participants' experience of using sliders when exploring possible events and their overall experience with transitory search. This includes investigation of the participants' satisfaction with the results returned by adjusting the slider settings and the participants' enjoyment when using the app.

\section{Findings and Discussion}

During the evaluations it was clear that participants were unsure about how the system worked to start with, but quickly learned how the sliders worked by playing around with them. Most users were a little confused to begin with about how to interpret the results when using the sliders and misunderstood the "bubbling" behaviour of the transient list of events. After using the app during the first task, they got a better understanding of how they could adjust the sliders to explore possible events. All participants found the sliders to be helpful in describing the events, as they were able to combine the information from outcomes with the current slider settings. Also, they 
found the sliders useful, as a way of searching for new events. One participant stated:

"I think, yeah, in general, I think they are much more helpful than I'm used to with apps. That's exactly what I am asking when I am looking for an event. So yeah, they are really handy"

\section{Interpretation of slider labels}

There were some problems regarding the labels (continuums) chosen for the sliders. In particular, participants found it difficult to understand the From far away $\leftrightarrow$ From here slider. They had different initial expectations of the results based on their slider settings, but were able to understand the reasoning behind the categorizations after a little use. This is interesting as it shows the participants, to some extent, were able to change their interpretation of the slider labels to better match the results returned. When developing slider labels it is thus necessary to test how users understand the slider labels, or to provide some flexibility in the slider values and labels by giving them the ability to work with a personalised sub-set of a larger set of available sliders.

Sliders middle has different meanings Putting a handle in the middle of the slider conveyed different meanings depending on the users' understanding of the slider labels. If they didn't understand what the slider meant, as with the slider From far away $\leftrightarrow$ From here, or the sliders did not matter to them, people thought that putting the handle in the middle neutralized it so that it did not influence the results. Conversely, if they had a good understanding of what events might fit in the middle of the slider, they utilized more of the slider spectrum, including the middle. One participant put the slider for entertaining/serious in the middle and said:

"I'll take something where I can get a bit inspired. That would be good. So I am taking a middle thing between entertaining and serious stuff. I don't want to hear about heart diseases, but still, I don't want to see a circus"

Afterwards the same participant considered the From far away $\leftrightarrow$ From here slider, saying, "I'm not sure that matters?" and proceeded to set the slider in the middle. As this behavior was exhibited by all participants, it was clear that the sliders are used differently depending on the users understanding of the slider. This underlines the importance of choosing understandable slider labels. Furthermore it shows, that users should be able to nullify specific sliders when exploring.

Fear of missing out

All participants mentioned a fear of missing out on events. This fear is articulated in two situations. The first is if the user thinks the sliders work as filters, thereby only returning a subset of events. One participant said:

"But I can't really figure out if all the events are here (on the list) all the time or it sorts something out. Like, I'm always very afraid of missing out on anything"

The second is if the user suspects that the events may not be tagged correctly, so relevant events may hide somewhere in the result list. Two different users made the following comments about this: 
"I always go through the (entire) list ... I want to be sure I'm not missing an important event"

"There could be a cool event down at the bottom, because one of the sliders are wrong"

This concern was also identified by Pearce et al. [11]. They suggested that it could indicate a lack of trust in the system, as participants fear it does not show them all relevant events. To avoid this issue the user needs to understand, through effective interaction design, that no results are removed from the list and that the most relevant results are at the top. This may be achieved by adding animations to show the users that the list is merely reordering. Alternatively, a brief introduction to the app could suffice. The fear of missing out on events could also be mitigated over time, as the user gains trust in the tagging of the events. To improve tagging, it might be useful to let the users contribute to the tagging, creating a more democratic classification of events.

\section{Serendipitous discoveries}

Several participants mentioned that the transitory search approach helped them discover an event that they had not considered looking for at the beginning. When trying to find an event for colleagues, one participant carefully adjusted the sliders and said:

"Yeah, I didn't think about a dining event, but now that I see it, it comes to mind that it could be a really nice instance to get to know each other. Yeah, maybe wine tastings too..."
It was clear that transitory search using the sliders was most useful when the participants were not trying to find specific events, or specific categories of events they had in mind. When searching for specific events or specific types of events, the transitory search paradigm was not so useful in returning satisfying results. This supports findings in $[9,10]$.

\section{Conclusions}

This paper has presented a prototype system for exploring urban events using transitory search on a smartphone. A user evaluation showed that the mobile platform could support in-time and in-place serendipitous event discovery. We also confirmed previously identified problems with the need to assure the user that no relevant information is hidden. We can confirm from our study that sliders as an interaction mechanism for facilitating serendipitous discoveries can be effectively used on a mobile platform. In addition, the evaluation showed that the middle of sliders might convey different meanings and leads users to use the middle of the slider differently under different circumstances as a result. The questions of providing appropriate slider labels, distrust in tagging and top results, and setting a slider to the middle as a meaningful action, need to be investigated further.

\section{References}

[1] Adomavicius, G. and Tuzhilin, A. Toward the next generation of recommender systems: a survey of the state-of-the-art and possible extensions, Knowledge and Data Engineering, 17 (2005), 734-749.

[2] Ayers, P. and Youssef, A. Investigating the influence of transitory information and motivation during instructional animations. Proc. ICLS 2008, ISLS (2008), 68-75. 
[3] Bellotti, V., Begole, B., Chi, E. H., Ducheneaut, N., Fang, J., Isaacs, E., et al. Activity-based serendipitous recommendations with the Magitti mobile leisure guide, Proc. CHI 2008, ACM (2008), 1157-1166.

[4] Cheverst, K., Davies, N., Mitchell, K., Friday, A. and Efstratiou, C. Developing a context-aware electronic tourist guide: some issues and experiences. Proc. $\mathrm{CHI}$ 2000, ACM (2000), 17-24.

[5] Forsblom, A., Nurmi, P., Åman, P. and Liikkanen, L. Out of the bubble: serendipitous even

recommendations at an urban music festival. Proc. IUI 2012, ACM (2012), 253-256.

[6] Gavalas, D., Konstantopoulos, C., Mastakas, K. and Pantziou, G., Mobile recommender systems in tourism, Journal of Network and Computer Applications, 39 (2014), 319-333.

[7] Marchionini, G. Exploratory search: from finding to understanding. Communications of ACM, 49, 4 (2006), 41-46.

[8] Pearce, J., Chang, S., Alzougool, B., Kennedy,G., Ainley, M. and Rodrigues, S. Search or explore: do you know what you're looking for? Proc. OzCHI 2011, ACM (2011), 253-256.
[9] Pearce, J., Chang, S., Kennedy,G., Ely, R. B. W. and Ainley, $M$. Search and explore: more than one way to find what you want. Proc. OzCHI 2012, ACM (2012), 469-478.

[10] Pearce, J. and Pardo, S. To search or to explore -that is the question: a study in mindful engagement. Proc. OzCHI 2008, ACM (2008), 251-254.

[11] Pearce, J. and Pardo, S. So now you're ready to play: but with what? a system to encourage playfu exploration. Proc. HCIEd 2009, ACM (2009), 2-2.

[12] Ricci, F., Rokach, L., Shapira, B. and Kantor, P.B. Recommender Systems Handbook, Springer, 2011.

[13] Schaller, R., Harvey, M. and Elsweiler, D.

Entertainment on the go: finding things to do and see while visiting distributed events. Proc. IIiX 2012, ACM (2012), 90-99.

[14] van Setten, M., Pokraev, S. and Koolwaaij. J. Context-Aware Recommendations in the Mobile Tourist Application COMPASS. Proc. AH 2004, Springer (2004), 235-244. 